\title{
Enquêtes des plaintes relatives aux pratiques de prévention et de contrôle des infections par la santé publique en Ontario, de 2015 à 2018
}

\author{
G Cadieux*, C Brown², H Sachdeva ${ }^{3}$
}

\begin{abstract}
Résumé
Contexte : À la suite d'une mise à jour du Protocole provincial concernant les plaintes relatives aux pratiques de prévention et de contrôle des infections en 2015, les unités de santé publique de l'Ontario ont reçu le mandat d'enquêter sur des plaintes relatives aux pratiques de prévention et de contrôle des infections ( $\mathrm{PCl}$ ) dans divers milieux, y compris ceux au sein desquels travaillent des professionnels de la santé réglementés. Aucun système de surveillance n'existe pour les plaintes relatives aux pratiques de $\mathrm{PCl}$; on en sait donc peu sur leur occurrence. Des témoignages anecdotiques suggèrent une augmentation récente des plaintes relatives aux pratiques de $\mathrm{PCl}$ ayant causé une demande accrue quant aux ressources de santé publique.
\end{abstract}

Objectifs : Décrire l'occurrence des plaintes et des manquements relatifs aux pratiques de $\mathrm{PCl}$ en Ontario entre 2015 et 2018 ainsi que les interventions réalisées par la santé publique en réponse à ceux-ci.

Méthodes : Les unités de santé publique de l'Ontario ont fait l'objet d'un sondage quant à l'occurrence et aux principaux défis des enquêtes suite aux plaintes relatives aux pratiques de $\mathrm{PCl}$, au moyen de questions ouvertes et fermées. Ce sondage a été diffusé par l'intermédiaire de la liste de diffusion du Conseil des médecins hygiénistes de l'Ontario. La collecte de données a eu lieu du 4 au 28 février 2019. Des analyses statistiques descriptives et une analyse thématique des réponses en texte narratif ont eu lieu.

Résultats : Vingt et une unités de santé publique ont répondu, représentant un taux de réponse de $60 \%$; un moins grand nombre d'unités de santé publique répondantes comptaient une population de moins de 100000 habitants. L'étude a révélé que le nombre de plaintes relatives aux pratiques de $\mathrm{PCl}$ a augmenté d'un facteur de presque six, passant d'un total de 79 plaintes en 2015 à 451 plaintes en 2018. Les manquements relatifs aux pratiques de $\mathrm{PCl}$ ont presque triplés; 61 ayant été déclarés en 2015 et 168 en 2018. Bien qu'une variation du nombre de plaintes et de manquements relatifs aux pratiques de $\mathrm{PCl}$ a été relevée parmi les unités de santé publique, le manquement le plus courant relatif aux pratiques de $\mathrm{PCl}$ concernait le retraitement inadéquat de dispositifs réutilisable. Les principaux défis rencontrés lors d'enquêtes des plaintes relatives aux pratiques de $\mathrm{PCl}$ incluaient le manque d'expertise et de formation du personnel, l'augmentation de la charge de travail et des coûts, des incohérences interjuridictionnelles et un manque de directives claires.

Conclusion : Les plaintes et manquements relatives aux pratiques de $\mathrm{PCl}$ ont augmenté en Ontario depuis 2015, lorsque le ministère de la Santé et des Soins de longue durée a modifié le protocole concernant les plaintes relatives aux pratiques de $\mathrm{PCl}$. Les unités de santé publique ont indiqué les défis suivants : manque d'expertise et de formation, augmentation de la charge de travail, incohérences interjuridictionnelles et manque de directives claires. D'autres études confirmant ces résultats, l'élaboration de pratiques exemplaires permettant de faire face à ces défis ainsi que des interventions visant à prévenir les manquements en matière de pratiques de $\mathrm{PCl}$ seraient utiles. Une éventuelle surveillance des plaintes en matière de pratiques de $\mathrm{PCl}$, comme pour les maladies à déclaration obligatoire, par exemple, serait également utile.
Cette oeuvre est mise à la disposition selon les termes de la licence internationale Creative Commons Attribution 4.0

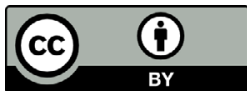

Affiliations

1 Santé publique Ottawa, Ottawa, Ontario (lorsque cette étude a été menée)

2 École d'épidémiologie et de santé publique, Université d'Ottawa, Ottawa, ON

${ }^{3}$ Toronto Public Health, Toronto, ON

${ }^{\star}$ Correspondance: genevieve. cadieux.ccsmt|@ssss.gouv.qc.ca 
Citation proposée : Cadieux G, Brown C, Sachdeva H. Enquête des plaintes relatives aux pratiques de prévention et de contrôle des infections par la santé publique en Ontario, de 2015 à 2018. Relevé des maladies transmissibles au Canada 2019;45(11):

318-25. https://doi.org/10.14745/ccdr.v45i11a03f

Mots-clés : prévention et contrôle des infections, manquement/faille en matière de contrôle des infections, cadre communautaire des soins de santé, pratiques/normes privées, procédures chirurgicales ambulatoires, prévention et en contrôle des infections croisées, contamination d'équipement

\section{Introduction}

De récentes enquêtes de santé publique en réponse à des plaintes relatives aux pratiques de prévention et de contrôle des infections (PCl) concernant des milieux de services personnels et des milieux de soins de santé communautaires en Ontario ont révélé des manquements significatifs aux pratiques exemplaires de retraitement des dispositifs médicaux, menant à des rappels et dépistages auprès des patients à grande échelle (1-3).

Contrairement aux maladies à déclaration obligatoire au niveau provincial, aucun système de surveillance provincial n'existe pour les plaintes et manquements relatifs aux pratiques de $\mathrm{PCl}$. Cependant, des témoignages anecdotiques suggèrent une demande accrue pesant sur les ressources des unités de santé publique, du fait d'une hausse du nombre de plaintes et de manquements relatifs aux pratiques de $\mathrm{PCl}$ ces dernières années, ainsi que de la complexité grandissante des enquêtes relatives aux pratiques de $\mathrm{PCl}$ (p. ex. faisant intervenir un vaste éventail de milieux de soins de santé et de service personnels ainsi que des procédures et équipements nouveaux).

Le présent article vise à décrire l'occurrence des plaintes et des manquements relatifs aux pratiques de $\mathrm{PCl}$ dans les milieux communautaires en Ontario entre 2015 et 2018 ainsi que les interventions de la santé publique en lien avec ceux-ci.

\section{Contexte}

La Loi sur la protection et la promotion de la santé (LPPS) (4) définit l'organisation des unités de santé publique en Ontario. Il existe actuellement 35 unités de santé publique en Ontario : 21 indépendantes d'administrations municipales locales, sept départements de santé publique régionaux et sept unités de santé publique liées à l'administration d'une municipale à de pallier supérieur ou à une autre administration municipale (5). Les programmes et services de santé publique obligatoires sont définis dans les Normes de santé publique de l'Ontario (NSPO) (6) et dans les protocoles et directives associés. Le Protocole concernant les plaintes relatives aux pratiques de prévention et de contrôle des infections (7), mis à jour en 2015 , demande aux unités de santé publique d'enquêter sur les plaintes relatives aux pratiques de $\mathrm{PCl}$ dans divers milieux, y compris, sans s'y limiter, les milieux où sont rendus des services personnels ( $p$. ex. salons de manucure, salons de coiffure, salons de tatouage) ainsi que tous les milieux où travaillent les professionnels de la santé réglementés ( $p$. ex. infirmiers et infirmières, médecins, dentistes). Par exemple, sur 61 plaintes relatives aux pratiques de $\mathrm{PCl}$ que Santé publique Ottawa a enquêtées en 2018, 28 (46 \%) concernaient des cliniques médicales, sept (11\%) concernaient des cliniques dentaires et 26 (43\%) concernaient d'autres milieux (p. ex. services personnels, prestataires de services paramédicaux) (Communication personnelle, Jacqueline Willmore, Santé publique Ottawa, 8 juillet 2019).

Le Protocole concernant les plaintes relatives aux pratiques de prévention et de contrôle des infections, 2019 (7) demande aux unités de santé publique de recevoir les plaintes relatives aux pratiques de $\mathrm{PCl}$, de les évaluer et de prendre des mesures pour réduire le risque de transmission des maladies infectieuses. La réponse à une plainte inclue généralement une inspection par un ou une inspecteur(e) de la santé publique et/ou un ou une infirmier(ère). Ils utilisent des outils de vérification (grilles d'audit) de Santé publique Ontario (8) et les ressources figurant dans les documents de pratiques exemplaires relatives à la $\mathrm{PCl}$, provenant notamment du Comité consultatif provincial des maladies infectieuses (CCPMI) $(9,10)$, afin d'évaluer les écarts par rapport aux pratiques exemplaires relatives à la $\mathrm{PCl}$. Le Protocole concernant les plaintes relatives aux pratiques de prévention et de contrôle des infections définit un manquement aux pratiques de $\mathrm{PCl}$ comme « le non-respect des pratiques de prévention et de contrôle des infections, entraînant ainsi un risque de transmission de maladies infectieuses aux clients, aux personnes présentes ou aux membres du personnel par l'exposition de ces personnes à du sang, à des liquides organiques, à des sécrétions, à des excrétions, à des muqueuses, à des lésions cutanées ou encore à du matériel contaminé et à des articles souillés " (7). Une minorité des plaintes relatives aux pratiques de $\mathrm{PCl}$ faites aux unités de santé publique s'avère constituer des manquements aux pratiques de $\mathrm{PCl}$; la majorité indique des écarts d'application des pratiques exemplaires de $\mathrm{PCl}$ qui, selon une évaluation du risque, ne représentent pas un risque suffisant de transmission d'infection pour être considérés comme un manquement. Santé publique Ontario offre son soutien aux unités de santé publique qui doivent effectuer des évaluations de risque complexes.

Si le médecin hygiéniste ou la personne désignée par celuici détermine qu'il y a eu manquement aux pratiques de $\mathrm{PCl}$, cela doit être divulgué publiquement sur le site Web de l'unité de santé publique, conformément au Protocole concernant les déclarations relatives aux pratiques de prévention et de contrôle des infections (11). Si un exploitant (p. ex. une personne exploitant un milieu de services personnels ou un milieu de soins) ne coopère pas à l'enquête de la plainte relative aux pratiques de $\mathrm{PCl}$ ou ne met pas en place des mesures correctives dans les délais convenus, des mesures peuvent être prises en vertu de la LPPS. Si le médecin hygiéniste ou un inspecteur de santé publique crois qu'une menace à la santé existe, une ordonnance 
découlant de l'article 13 de la LPPS peut être utilisé pour faire cesser une pratique ou pour fermer un milieu de services personnels ou de soins.

\section{Méthodes}

\section{Contexte, conception et population de l'étude}

Un sondage portant sur l'occurrence de plaintes et de manquements relatifs aux pratiques de $\mathrm{PCl}$ dans les milieux communautaires en Ontario entre 2015 et 2018 et les interventions réalisées par la santé publique en réponse à ceux-ci a été mené pour contribuer aux discussions au cours d'un atelier d'une journée portant sur les enquêtes en lien avec les manquements aux pratiques de $\mathrm{PCl}$ dans des milieux de soins, tenu lors du Congrès de santé publique de l'Ontario en mars 2019. La population à l'étude était composée des 35 unités de santé publique ontariennes. Ce sondage a été diffusé au moyen de la liste de distribution du Conseil des médecins hygiénistes de l'Ontario le 4 février 2019 et a été suivi d'un rappel les 12 et 25 février 2019. La période de collecte des données a pris fin le 28 février 2019. Les répondants devaient avoir accès aux dossiers des plaintes relatives aux pratiques de $\mathrm{PCl}$ de leur unité de santé publique pour pouvoir répondre au sondage. Les réponses ont été directement saisies sur un formulaire CheckMarket ${ }^{\circledR}$ en ligne. Les données ont alors été transférées dans un fichier Microsoft Excel (2010; Redmond, Washington, États-Unis). Les caractéristiques démographiques des unités de santé publique (c.-à-d. la taille de la population, la densité de la population par kilomètre carré) fondées sur le Recensement de 2016 ont été obtenues auprès de Statistique Canada (12).

\section{Instrument de sondage}

Le sondage était composé d'une combinaison de questions ouvertes et fermées. Le sondage demandait aux répondants de sélectionner leur unité de santé publique et de fournir les renseignements suivants pour chaque année entre 2015 et 2018 : le nombre de plaintes relatives aux pratiques de $\mathrm{PCl}$ reçues et ayant fait l'objet d'une enquête, le nombre de manquements aux pratiques de $\mathrm{PCl}$, le nombre de manquements pour lesquels un rappel des patients a été effectué, le nombre de patients avisés lors d'un rappel, le nombre de manquements pour lesquels une transmission de virus de l'hépatite $B(\mathrm{VHB})$, de virus de l'hépatite $\mathrm{C}(\mathrm{VHC})$ ou de virus de l'immunodéficience humaine $(\mathrm{VIH})$ a été suspectée et le nombre de manquements pour lesquels une ordonnance a été émise en vertu de I'article 13 de la LPPS. Le sondage demandait aux répondants d'indiquer la ou les méthode(s) utilisée(s) pour aviser les patients pour chacun de leurs deux rappels les plus récents. Le sondage demandait également aux répondants de sélectionner, dans une liste fondée sur l'outil de vérification de Santé publique Ontario (5), les trois écarts aux pratiques exemplaires de PCI les plus fréquemment cités comme motif de la plainte, observés au cours des enquêtes suite à une plainte et relevés dans le cadre des manquements aux pratiques de $\mathrm{PCl}$. Enfin, les répondants étaient invités à décrire les trois principaux défis auxquels leur unité de santé publique avait été confrontée dans le cadre d'enquêtes de plaintes relatives aux pratiques de $\mathrm{PCl}$. La validité de l'instrument de sondage a été évaluée par des membres du comité organisateur de l'atelier d'une journée du Congrès de santé publique de l'Ontario. (L'instrument de sondage est disponible, en anglais seulement, sur demande auprès de l'auteur correspondant.)

\section{Analyse}

Une analyse descriptive des données quantitatives a été menée dans Microsoft Excel 2010 et les réponses aux questions ouvertes ont fait l'objet d'une analyse thématique. Le nombre de réponses pour l'année 2018 ayant été supérieur à celui de 2015; une analyse de sensibilité a été effectuée pour évaluer si les tendances temporelles observées découlaient des données manquantes. Les données provenant des répondants ayant fourni des données pour toutes les années entre 2015 et 2018 ont été comparées aux données de tous les répondants, y compris ceux pour lesquels des données manquaient.

\section{Résultats}

Vingt et une $(60 \%)$ des 35 unités de santé publique de l'Ontario ont participé au sondage. Le tableau 1 décrit les caractéristiques des unités de santé publique participantes et non participantes. Un moins grand nombre d'unités de santé publique participantes que non participantes comptaient une population inférieure à 100000 habitants et une densité de population supérieure à $300 / \mathrm{km}^{2}$.

\section{Tableau 1 : Caractéristiques des unités de santé publique ontariennes participantes et non participantes (selon les données du Recensement de 2016)}

\begin{tabular}{|c|c|c|c|c|c|c|}
\hline \multirow[t]{2}{*}{ Caractéristique } & \multicolumn{2}{|c|}{$\begin{array}{c}\text { Unités de } \\
\text { santé publique } \\
\text { participantes } \\
(\mathrm{N}=21)\end{array}$} & \multicolumn{2}{|c|}{$\begin{array}{l}\text { Unités de santé } \\
\text { publique non } \\
\text { participantes } \\
(\mathrm{N}=14)\end{array}$} & \multicolumn{2}{|c|}{$\begin{array}{c}\text { Toutes les } \\
\text { unités de } \\
\text { santé publique } \\
\text { de l'Ontario } \\
(\mathrm{N}=35)\end{array}$} \\
\hline & $n$ & $\%$ & $n$ & $\%$ & $n$ & $\%$ \\
\hline \multicolumn{7}{|c|}{ Taille de la population } \\
\hline$<100000$ & 2 & 9,5 & 3 & 21,4 & 5 & 14,3 \\
\hline 100 000-174999 & 7 & 33,3 & 5 & 35,7 & 12 & 34,3 \\
\hline $175000-499999$ & 7 & 33,3 & 2 & 14,3 & 9 & 25,7 \\
\hline$\geq 500000$ & 5 & 23,8 & 4 & 28,6 & 9 & 25,7 \\
\hline \multicolumn{7}{|c|}{ Densité de la population par km² } \\
\hline$<10$ & 5 & 23,8 & 3 & 21,4 & 8 & 22,9 \\
\hline $10-39,9$ & 6 & 28,6 & 4 & 28,6 & 10 & 28,6 \\
\hline $40-299,9$ & 6 & 28,6 & 3 & 21,4 & 9 & 25,7 \\
\hline$\geq 300$ & 4 & 19,0 & 4 & 28,6 & 8 & 22,9 \\
\hline
\end{tabular}


Globalement, le nombre de plaintes relatives aux pratiques de $\mathrm{PCl}$ a augmenté d'un facteur de 5,7 parmi les unités de santé publique ontariennes participantes, passant de 79 en 2015 à 451 en 2018 (tableau 2). Le nombre de manquements aux pratiques de $\mathrm{PCl}$ a augmenté d'un facteur de 2,8, passant de 61 en 2015 à 168 en 2018; la proportion des plaintes relatives aux pratiques de $\mathrm{PCl}$ déterminées comme étant des manquements a cependant diminué, passant de $77 \%$ en 2015 à $37 \%$ en 2018 (tableau 2). II convient de noter que le nombre d'unités de santé publique déclarant ces renseignements a été supérieur pour l'année 2018 (21/35, 60 \%) que pour l'année 2015 (14/35, $40 \%$ ). Pour évaluer si la hausse observée du nombre de plaintes et de manquements relatifs aux pratiques de $\mathrm{PCl}$ pouvait être attribuée au plus grand nombre d'unités de santé publique déclarant des données pour 2018 que pour 2015, une analyse de sensibilité a été menée. Parmi les 14 unités de santé publique ayant déclaré des données pour les quatre années, le nombre de plaintes relatives aux pratiques de $\mathrm{PCl}$ a augmenté d'un facteur de 5,1 (passant de 79 en 2015 à 405 en 2018) et le nombre de manquements a augmenté d'un facteur de 2,6 (passant de 61 en 2015 à 157 en 2018); ces résultats sont presqu'identiques à ceux incluant les unités de santé publique pour lesquelles des données manquent.

Les données du tableau 2 révèlent également qu'environ $90 \%$ de toutes les plaintes relatives aux pratiques de $\mathrm{PCl}$ avaient donné lieu à au moins une inspection; cette proportion est demeurée stable au cours du temps. Entre 2015 et 2018 , $15(2,8 \%)$ des 538 manquements aux pratiques de $\mathrm{PCl}$ ont été évalués comme posant un risque de transmission d'infection suffisant pour nécessiter un rappel et dépistage des patients. Le plus fréquemment, les patients ont été avisés par lettre
(7/10 unités de santé publique), communiqué de presse ou communiqué d'intérêt public (6/10 unités de santé publique) et/ou par une publication sur le site Web de l'unité de santé publique (6/10 unités de santé publique). Le nombre de manquements aux pratiques de $\mathrm{PCl}$ avec transmission suspectée de virus VHB ou VHC a été faible (4/538 manquements; 0,7\%) et aucune transmission suspectée de $\mathrm{VIH}$ n'a été relevée. La proportion de manquements aux pratiques de $\mathrm{PCl}$ pour lesquels une ordonnance en vertu de l'article 13 de la LPPS a été émise a diminué au cours du temps, passant de $23 \%$ en 2015 à $10 \%$ en 2018.

Une variation au niveau du nombre de plaintes et de manquements relatifs aux pratiques de $\mathrm{PCl}$ a été observée entre les unités de santé publique (tableau 3 ). Cette variation ne s'explique pas uniquement par la taille de la population des unités de santé publique; le nombre de plaintes relatives aux pratiques de $\mathrm{PCl}$ pour 100000 habitants est de 0,9 à 62,1 (médiane : 9,4; moyenne : 12,9).

Les types d'écarts par rapport aux pratiques exemplaires de $\mathrm{PCl}$ les plus fréquemment cités comme motif de plainte étaient facilement observables par les clients/patients : équipement sale, environnement sale et absence d'hygiène des mains (Table 4). Le retraitement inadéquat d'équipement réutilisable et les politiques et procédures de $\mathrm{PCl}$ non-existantes ou inadéquates constituaient les écarts les plus courants relevés au cours de l'enquête sur les plaintes. Les écarts les plus fréquents relevés quant aux manquements étaient le retraitement inadéquat d'équipement réutilisable, l'utilisation inadéquate de flacons ou de solutions multidose et des équipements de protection individuelle manquants ou inadéquats.

\section{Tableau 2 : Nombre total de plaintes et de manquements relatifs aux pratiques de prévention et de contrôle des infections $(\mathrm{PCl})$ ayant fait l'objet d'une enquête par les unités de santé publique ontariennes participantes, entre 2015 et 2018}

\begin{tabular}{|c|c|c|c|c|c|c|c|c|c|c|}
\hline \multirow[t]{2}{*}{ Plaintes et manquements } & \multicolumn{2}{|c|}{$\begin{array}{c}2015 \\
(\mathrm{~N}=14 \text { USP })\end{array}$} & \multicolumn{2}{|c|}{$\begin{array}{c}2016 \\
(\mathrm{~N}=16 \text { USP) }\end{array}$} & \multicolumn{2}{|c|}{$\begin{array}{c}2017 \\
(\mathrm{~N}=19 \text { USP })\end{array}$} & \multicolumn{2}{|c|}{$\begin{array}{c}2018 \\
(\mathrm{~N}=21 \text { USP) }\end{array}$} & \multicolumn{2}{|c|}{ Total: $\mathbf{2 0 1 5 - 2 0 1 8}$} \\
\hline & $n$ & $\%$ & $n$ & $\%$ & $\mathbf{n}$ & $\%$ & $n$ & $\%$ & $\mathbf{n}$ & $\%$ \\
\hline $\begin{array}{l}\text { Nombre total de plaintes relatives aux pratiques de } \mathrm{PCl}^{\mathrm{a}} \\
\text { reçues }\end{array}$ & 79 & 100,0 & 189 & 100,0 & 366 & 100,0 & 451 & 100,0 & 1085 & 100,0 \\
\hline avec $\geq 1$ inspection & 71 & 89,9 & 170 & 89,9 & 340 & 92,9 & 414 & 91,8 & 995 & 91,7 \\
\hline Nombre total de manquements aux pratiques de $\mathrm{PCl}$ & 61 & 77,2 & 108 & 57,1 & 201 & 54,9 & 168 & 37,3 & 538 & 49,6 \\
\hline avec rappel des patients & 2 & 3,3 & 1 & 0,9 & 6 & 3,0 & 6 & 3,6 & 15 & 2,8 \\
\hline avec transmission suspectée de VHB ou VHC & 1 & 1,6 & 0 & 0 & 2 & 1,0 & 1 & 0,6 & 4 & 0,7 \\
\hline $\begin{array}{l}\text { ayant fait l'objet d'une ordonnance en vertu de l'article } 13 \text { de } \\
\text { la LPPS }\end{array}$ & 14 & 23,0 & 16 & 14,8 & 25 & 12,4 & 17 & 10,1 & 72 & 13,4 \\
\hline Nombre total de patients avisés lors d'un rappel & 570 & - & $N / S$ & - & 703 & - & 5112 & - & 6385 & - \\
\hline
\end{tabular}

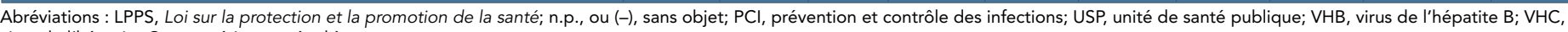
virus de l'hépatite $C_{i} \geq$, supérieur ou égal à

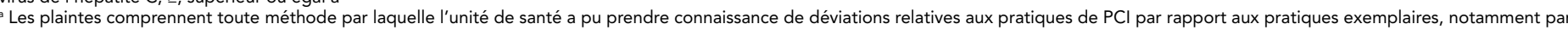
un cas de maladie à déclaration obligatoire ou un rapport d'épidémie ou une inspection routinière d'un environnement de services personnels 
Tableau 3 : Nombre de plaintes et de manquements relatifs aux pratiques de prévention et contrôle des infections (PCl) ayant fait l'objet d'une enquête, par unité de santé publique ontarienne participante, entre 2015 et 2018

\begin{tabular}{|c|c|c|c|c|c|c|c|c|c|c|c|c|c|c|c|}
\hline \multirow[b]{2}{*}{ Plaintes et manquements } & \multicolumn{3}{|c|}{$\begin{array}{c}2015 \\
(\mathrm{~N}=14 \text { USP })\end{array}$} & \multicolumn{3}{|c|}{$\begin{array}{c}2016 \\
(N=16 \text { USP })\end{array}$} & \multicolumn{3}{|c|}{$\begin{array}{c}2017 \\
(\mathrm{~N}=19 \text { USP })\end{array}$} & \multicolumn{3}{|c|}{$\begin{array}{c}2018 \\
(\mathrm{~N}=21 \text { USP })\end{array}$} & \multicolumn{3}{|c|}{ Total: 2015 à 2018} \\
\hline & 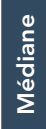 & 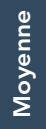 & 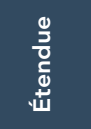 & 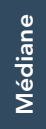 & 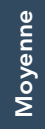 & 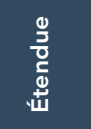 & 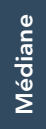 & 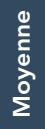 & $\begin{array}{l}\frac{9}{0} \\
\frac{\pi}{2}\end{array}$ & $\begin{array}{l}\frac{\Phi}{c} \\
\frac{c}{\pi} \\
\frac{0}{0} \\
\Sigma \\
\Sigma\end{array}$ & 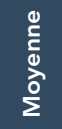 & 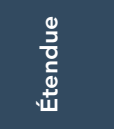 & 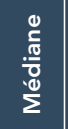 & 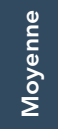 & 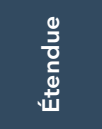 \\
\hline Nombre de plaintes relatives aux pratiques de $\mathrm{PCl}^{\mathrm{a}}$ reçues & 2 & 6 & 3 à 36 & 5 & 12 & 0 à 71 & 5 & 19 & 0 à 90 & 9 & 21 & 1 à 130 & 13 & 52 & 2 à 278 \\
\hline avec $\geq 1$ inspection & 1 & 5 & 0 à 36 & 3 & 11 & 0 à 71 & 5 & 19 & 0 à 89 & 8 & 20 & 1 à 130 & 12 & 47 & 2 à 274 \\
\hline Nombre de manquements aux pratiques de $\mathrm{PCl}$ & 0 & 5 & 0 à 36 & 0 & 7 & 0 à 71 & 1 & 11 & 0 à 89 & 1 & 8 & 0 à 95 & 2 & 26 & 0 à 274 \\
\hline avec rappel des patients & 0 & 0 & 0 à 1 & 0 & 0 & 0 à 1 & 0 & 0 & 0 à 1 & 0 & 0 & 0 à 2 & 0 & 1 & 0 à 3 \\
\hline avec transmission suspectée de VHB ou VHC & 0 & 0 & 0 à 1 & 0 & 0 & 0 & 0 & 0 & 0 à 1 & 0 & 0 & 0 à 1 & 0 & 0 & 0 à 1 \\
\hline $\begin{array}{l}\text { ayant fait l'objet d'une ordonnance ordre en vertu de I' } \\
\text { de la LPPS }\end{array}$ & 0 & 1 & 0 à 12 & 0 & 1 & 0 à 11 & 0 & 1 & 0 à 11 & 0 & 1 & 0 à 4 & 1 & 3 & 0 à 31 \\
\hline Nombre de patients avisés lors d'un rappel & 0 & 48 & 0 à 540 & 0 & 0 & 0 à $N / S$ & 0 & 47 & 0 à 500 & 0 & 301 & 0 à 4,600 & 0 & 304 & 0 à 5,140 \\
\hline
\end{tabular}

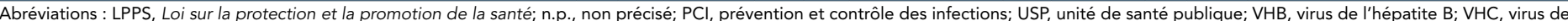
I'hépatite $C_{i} \geq$, supérieur ou égal à

a Les plaintes incluent toute méthode par laquelle l'unité de santé a pu prendre connaissance de déviations relatives aux pratiques de PCl par rapport aux pratiques exemplaires, notamment par

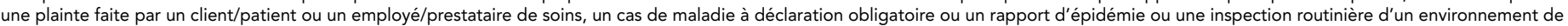
services personnels

Tableau 4 : Les trois types d'écarts des pratiques exemplaires ${ }^{\mathrm{a}}$ de $\mathrm{PCl}$ les plus fréquemment relevés par les unités de santé publique ontariennes participantes $(\mathrm{N}=21)$

\begin{tabular}{|c|c|c|}
\hline \multirow{2}{*}{$\begin{array}{c}\text { Les trois types d'écarts des pratiques } \\
\text { exemplaires de } \mathrm{PCl} \text { les plus } \\
\text { fréquemment relevés }\end{array}$} & \multicolumn{2}{|c|}{$\begin{array}{l}\text { Nombre d'unités } \\
\text { de santé } \\
\text { publique }\end{array}$} \\
\hline & $\mathrm{n}$ & $\%$ \\
\hline \multicolumn{3}{|l|}{ Cités comme le motif de la plainte initiale } \\
\hline $\begin{array}{l}\text { Retraitement de l'équipement ou du matériel } \\
\text { médical utilisé pour dispenser les soins aux } \\
\text { patients ou clients }\end{array}$ & 20 & 95,2 \\
\hline $\begin{array}{l}\text { Nettoyage de l'environnement en milieu de } \\
\text { soins de santé, c.-à-d. les aires où les soins sont } \\
\text { fournis }\end{array}$ & 10 & 47.6 \\
\hline Hygiène des mains & 7 & 33,3 \\
\hline \multicolumn{3}{|l|}{ Relevés au cours de l'enquête sur la plainte } \\
\hline $\begin{array}{l}\text { Retraitement de l'équipement ou du matériel } \\
\text { médical utilisé pour dispenser les soins aux } \\
\text { patients ou clients }\end{array}$ & 16 & 76,2 \\
\hline Politiques et procédures générales & 10 & 47,6 \\
\hline $\begin{array}{l}\text { Nettoyage général de l'environnement, y } \\
\text { compris les produits utilisés }\end{array}$ & 6 & 28,6 \\
\hline Formation & 6 & 28,6 \\
\hline \multicolumn{3}{|l|}{ Relevés lors d'un manquement aux pratiques de $\mathrm{PCl}$} \\
\hline $\begin{array}{l}\text { Retraitement de l'équipement ou du matériel } \\
\text { médical utilisé pour dispenser les soins aux } \\
\text { patients ou clients }\end{array}$ & 18 & 85,7 \\
\hline $\begin{array}{l}\text { Flacons ou solutions de médicaments } \\
\text { injectables }\end{array}$ & 6 & 28,6 \\
\hline Équipement de protection individuelle (ÉPI) & 4 & 19,0 \\
\hline
\end{tabular}

Abréviation : $\mathrm{PCl}$, prévention et contrôle des infections

a Basé sur la Liste de vérification pour la $\mathrm{PCl}$ dans le cadre de la pratique en cabinet et en clinique - Éléments essentiels de Santé publique Ontario (8)

Le tableau 5 décrit les thèmes relevés dans les réponses des répondants à la question « Décrivez les trois principaux défis auxquels votre unité de santé publique a été confrontée lors de l'enquête des plaintes relatives aux pratiques de $\mathrm{PCl}$. » Les trois principaux thèmes relevés ont été le manque d'expertise ou de formation au sein du personnel de l'unité de santé publique (60\%), une augmentation de la charge de travail et/ou des coûts découlant des enquêtes sur les plaintes ( $55 \%$ ) et des

Tableau 5 : Principaux défis rencontrés lors de l'enquête des plaintes relatives aux pratiques de prévention et contrôle des infections $(\mathrm{PCl})$ rapportés par les unités de santé publique ontariennes participantes $(N=20)$

\begin{tabular}{|l|r|r|}
\hline \multicolumn{1}{|c|}{ Thèmes relevés } & \multicolumn{2}{c|}{$\begin{array}{c}\text { Unités } \\
\text { de santé } \\
\text { publique }\end{array}$} \\
\cline { 2 - 3 } & $\mathrm{n}$ & \multicolumn{1}{c|}{$\%$} \\
\hline $\begin{array}{l}\text { Manque d'expertise ou de formation au sein du } \\
\text { personnel de l'unité de santé publique }\end{array}$ & 12 & 60,0 \\
\hline $\begin{array}{l}\text { Augmentation de la charge de travail et/ou des coûts } \\
\text { découlant des enquêtes sur les plaintes relatives aux } \\
\text { pratiques de PCl }\end{array}$ & 11 & 55,0 \\
\hline $\begin{array}{l}\text { Incohérences dans les enquêtes sur les plaintes } \\
\text { relatives aux pratiques de PCl parmi les unités de santé } \\
\text { publique }\end{array}$ & 7 & 35,0 \\
\hline $\begin{array}{l}\text { Manque de directives claires quant à l'enquête } \\
\text { des plaintes relatives aux pratiques de PCl et à la } \\
\text { divulgation des manquements aux pratiques de PCl }\end{array}$ & 6 & 30,0 \\
\hline $\begin{array}{l}\text { Manque de soutien et/ou rôle ambigu des ordres } \\
\text { professionnels provinciaux en soins de santé }\end{array}$ & 6 & 30,0 \\
\hline $\begin{array}{l}\text { Exécution/obtention des évaluations du risque de } \\
\text { transmission d'infection pour les manquements aux } \\
\text { pratiques de PCl }\end{array}$ & 5 & 25,0 \\
\hline $\begin{array}{l}\text { Gestion des exploitants des milieux ciblés par une } \\
\text { enquête de plainte relative aux pratiques de PCl }\end{array}$ & 5 & 25,0 \\
\hline Questions d'ordre juridique & 3 & 15,0 \\
\hline Divulgation au public et perception du public ${ }^{b}$ & 3 & 15,0 \\
\hline
\end{tabular}

Abréviation : $\mathrm{PCl}$, prévention et contrôle des infections

a Exemples de défis juridiques : « responsabilité juridique dans le cadre de divulgations de manquements et de rappels des patients ( $p$. ex. le médecin hygiéniste ne va pas dans le même sens que l'évaluation du risque de Santé publique Ontario, une unité de santé publique évaluant des éléments à 'risque élevé' dans les listes de vérification de Santé publique Ontario comme des risques moins élevés que leur description dans la liste de vérification dans le contexte de l'enquête globale) » et « responsabilités juridiques assumées par les unités de santé publique dans le cadre d'un rappel des patients "

bxemple de défis relatifs à la divulgation au public : " gestion de la divulgation au public : quel niveau de détail, moment opportun du communiqué de presse, formulation [forum aux questions]» 
incohérences dans les enquêtes sur les plaintes parmi les unités de santé publique (35\%). Selon un répondant, ce dernier point était « en particulier un problème lorsque le professionnel de la santé a des bureaux dans plusieurs unités de santé publique ». Un manque de directives claire quant à l'enquête des plaintes relatives aux pratiques de $\mathrm{PCl}$ et à la divulgation des manquements aux pratiques de $\mathrm{PCl}$ a été relevé comme un défi par $30 \%$ des répondants. Des exemples incluent « définir les médi-spas et établir une approche claire quant aux enquêtes sur les manquements aux pratiques de $\mathrm{PCl}$ dans les médispas » et un " manque de directives claires permettant de déterminer quand la divulgation et/ou le rappel des patients est nécessaire ».

\section{Discussion}

Notre sondage auprès des unités de santé publique de l'Ontario a été le premier à montrer une augmentation d'un facteur de presque six du nombre de plaintes relatives aux pratiques de $\mathrm{PCl}$ et presque un triplement du nombre de manquements aux pratiques de $\mathrm{PCl}$ dans les milieux de soins de santé et de services personnels communautaires entre 2015 et 2018 . On a évalué qu'une faible proportion $(2,8 \%)$ des manquements aux pratiques de $\mathrm{PCl}$ posait un risque de transmission d'infection suffisant pour nécessiter un rappel des patients et un dépistage du VHB, VHC et $\mathrm{VIH}$. Une grande variation a été observée dans le nombre de plaintes et de manquements parmi les unités de santé publique; cette variation ne s'explique pas uniquement du fait de la taille de la population. Les manquements aux pratiques de $\mathrm{PCl}$ ayant fait l'objet d'une enquête par les unités de santé publique concernaient le plus fréquemment le retraitement inadéquat d'équipement réutilisable. Les défis des enquêtes sur les plaintes relatives aux pratiques de $\mathrm{PCl}$ les plus fréquemment rapportés par les unités de santé publique incluaient le manque d'expertise ou de formation du personnel, l'augmentation de la charge de travail du personnel et des coûts, des incohérences entre les unités de santé publique et le manque de directives claires pour soutenir la cohérence des enquêtes et des interventions de santé publique.

Nous n'avons connaissance d'aucune autre étude publiée décrivant les tendances temporelles des plaintes ou des manquements relatifs aux pratiques de $\mathrm{PCl}$ dans les milieux de services personnels ou de soins de santé communautaires au Canada ou ailleurs. Les données sur les infections de VBH et VCH associées aux soins de santé des Centers for Disease Control and Prevention (CDC) suggèrent que dans des milieux de soins de santé (autres que des hôpitaux), le nombre d'éclosions de VHB associées aux soins de santé était le plus élevé en 2010 (en lien avec les manquements aux pratiques de $\mathrm{PCl}$ relatifs aux tests de glycémie au chevet du patient dans les centres de soins de longue durée) et le nombre d'éclosions de VHC associées aux soins de santé était le plus élevé en 2015 (en lien avec des manquements aux pratiques de
$\mathrm{PCl}$ dans des services ambulatoires d'hémodialyse ) (13). Ces données, cependant, reflètent uniquement les manquements aux pratiques de $\mathrm{PCl}$ dans le cadre desquels la transmission du VHB ou du VHC était probablement liée à une éclosion (c.-à-d. au moins deux cas) et uniquement dans des milieux de soins de santé. L'occurrence des manquements aux pratiques de $\mathrm{PCl}$ dans les milieux de soins de santé et de services personnels communautaires est probablement influencée par plusieurs facteurs, notamment la complexité et la diversité croissantes des services fournis, l'entrée sur le marché de nouveaux types de dispositifs réutilisables nécessitant un retraitement et la capacité d'adaptation rapide des organismes de réglementation, des programmes de formation et des prestataires de services à ces changements afin d'assurer une prestation de services sécuritaire. Une explication possible de l'augmentation observée des plaintes relatives aux pratiques de $\mathrm{PCl}$ en Ontario est la sensibilisation et la déclaration accrues des problèmes de $\mathrm{PCl}$ par les clients/patients et prestataires de services; cette prise de conscience accrue pourrait être liée à quelques manquements aux pratiques de $\mathrm{PCl}$ très médiatisés (1-3).

Une force de ce sondage a été la possibilité d'étudier les tendances des occurrences de plaintes et de manquements relatifs aux pratiques de $\mathrm{PCl}$ au fil du temps. Même si l'instrument de sondage a fait l'objet d'une vérification de validité, il n'a pas été soumis à une évaluation officielle de la fiabilité. Notre taux de participation au sondage a été relativement élevé, mais moins d'unités de santé publique avec une faible population et une densité de population très élevée ont participé; par conséquent, nos résultats peuvent ne pas être représentatifs de l'ensemble de l'Ontario. Une autre limite de notre étude a été les données manquantes pour certaines unités de santé publique pour les années de 2015 à 2017; cependant, une analyse de sensibilité a démontré que notre conclusion d'augmentation des plaintes et des manquements relatifs aux pratiques de $\mathrm{PCl}$ au fil du temps se maintenait lorsque l'analyse était limitée aux unités de santé publique ayant fourni des données pour toutes les années. Notre instrument de sondage n'a pas recueilli de données sur les plaintes relatives aux pratiques de $\mathrm{PCl}$ touchant les milieux de soins de santé communautaires séparément des plaintes touchant les milieux de services personnels. Notre enquête a été limitée par le manque de directives détaillées relativement à l'enquête des plaintes relatives aux pratiques de $\mathrm{PCl}$; par conséquent, les données recueillies dans le cadre de ce sondage reflètent la variation dans l'interprétation et l'application du Protocole concernant les plaintes relatives aux pratiques de prévention et de contrôle des infections (7) et du Protocole concernant les déclaration en matière de pré vention et de contrôle des infections (11) de l'Ontario. De plus, notre sondage se fonde sur les plaintes relatives aux pratiques de $\mathrm{PCl}$ et sous-estime ainsi probablement le réel fardeau des écarts par rapport aux pratiques exemplaires et des manquements aux pratiques de $\mathrm{PCl}$. 
D'autres études sont nécessaires pour confirmer nos résultats et pour évaluer si cette augmentation des plaintes et des manquements relatifs aux pratiques de $\mathrm{PCl}$ observée en Ontario s'observe également dans d'autres provinces. Idéalement, l'occurrence des plaintes et des manquements relatifs aux pratiques de $\mathrm{PCl}$ serait suivie de façon routinière par un système de surveillance provincial similaire à celui utilisé pour les maladies à déclaration obligatoire; un tel système aiderait la santé publique à remplir son mandat de protection de la santé et permettrait de réaliser des études. Notre sondage souligne qu'un manque de formation et d'expertise, une hausse de la charge de travail, des incohérences interjuridictionnelles et un manque de directives claire sont des défis importants auxquels font face les unités de santé publique dans le cadre d'enquêtes de plaintes relatives aux pratiques de $\mathrm{PCl}$.

Des communautés de pratique portant sur l'enquête des plaintes relatives aux pratiques de $\mathrm{PCl}$ par la santé publique ont été établies en Ontario pour aider à faire face à certains de ces défis. De plus, depuis 2018, un groupe de travail du Conseil des médecins hygiénistes de l'Ontario examine des façons d'améliorer la cohérence opérationnelle des unités de santé publique menant des enquêtes sur des plaintes relatives aux pratiques de $\mathrm{PCl}$. Dans ce contexte, nous avons effectué une analyse environnementale des directives et des documents de soutien relatifs aux enquêtes sur les manquements aux pratiques de $\mathrm{PCl}$. Nous n'avons trouvé de directives explicites accessibles publiquement qu'auprès de deux organismes de santé publique. Les lignes directrices au Québec se limitent aux manquements en matière de retraitement des dispositifs médicaux, sont applicables à des environnements hospitaliers et ont recours à une approche d'évaluation du risque quantitative ou semi-quantitative $(14,15)$. En revanche, des lignes directrices des CDC sont applicables à un plus vaste éventail de situations et avaient recours à une approche d'évaluation du risque qualitative (16). (Résultats complets de l'analyse disponibles, en anglais seulement, sur demande.) Des études additionnelles sont nécessaires pour déterminer les meilleures méthodes pour enquêter les plaintes relatives aux pratiques de $\mathrm{PCl}$ et pour gérer les manquements aux pratiques de $\mathrm{PCl}$; les résultats de ces études devraient aider à élaborer des directives et des outils clairs et cohérents. Des études sont également nécessaires afin d'identifier des interventions efficaces permettant d'éviter ou de réduire l'occurrence des manquementsaux pratiques de $\mathrm{PCl}$ dans les milieux communautaires.

\section{Conclusion}

Les plaintes et les manquements relatifs aux pratiques de $\mathrm{PCl}$ ont augmenté en Ontario depuis 2015. Les unités de santé publique ont rapportés le manque d'expertise et de formation, l'augmentation de la charge de travail, des incohérences interjuridictionnelles et le manque de directives claires comme principaux défis rencontrés lors d'enquêtes sur les plaintes relatives aux pratiques de $\mathrm{PCl}$. De plus amples recherches confirmant ces résultats, l'élaboration de pratiques exemplaires permettant de faire face à ces défis ainsi que des interventions visant à prévenir les manquements aux pratiques de $\mathrm{PCl}$ seraient utiles. Une éventuelle surveillance des plaintes relatives aux pratiques de $\mathrm{PCl}$, comme pour les maladies à déclaration obligatoire, par exemple, serait également utile.

\section{Déclaration des auteurs}

G. C. a élaboré et analysé le sondage, a contribué à l'analyse environnementale et a été l'auteur principal du manuscrit. C. B. a effectué l'analyse environnementale et a fourni des commentaires sur le manuscrit. H. S. a supervisé l'analyse environnementale et a fourni des commentaires sur le manuscrit.

\section{Conflit d'intérêts}

Aucun.

\section{Remerciements}

Les auteurs aimeraient remercier les unités de santé publique de l'Ontario qui ont participé au sondage, Santé publique Ontario, le ministère de la Santé et des Soins de longue durée de l'Ontario et le Laboratoire de Santé publique Ontario pour leur soutien aux enquêtes sur les plaintes et les manquements relatifs à la prévention et au contrôle des infections. Les auteurs aimeraient également remercier J. Beehler, bibliothécaire de la santé publique, pour son aide dans la mise en œuvre de l'analyse environnementale, ainsi que les organismes de santé publique ayant participé à l'analyse environnementale.

\section{Financement}

Les auteurs ont reçu une aide salariale de leur organisation respective pour effectuer ce travail.

\section{Références}

1. Willmore J, Ellis E, Etches V, Labrecque L, Osiowy C, Andonov A, McDermaid C, Majury A, Achonu C, Maher M, MacLean B, Levy I. Public health response to a large-scale endoscopy infection control lapse in a nonhospital clinic. Can J Infect Dis Med Microbiol 2015 Mar-Apr;26(2):77-84. DOl PubMed

2. Ottawa Public Health. Infection prevention and control lapse at Main Street Family Medical Centre. Ottawa (ON): Ottawa Public Health; 2019 (Accédé 2019-06-08). http:// www.ottawapublichealth.ca/en/public-health-services/ ipac-lapse-2018.aspx 
3. Middlesex-London Health Unit. Infection prevention \& control lapse at ParaMed Clinics. London (ON): Middlesex-London Health Unit; 2018 Aug 20.

https://www.healthunit.com/ipac-lapse-paramed

4. Le gouvernement de l'Ontario. Loi sur la protection et la promotion de la santé, L.R.O. 1990, chap. H.7, Ontario, (1990). Toronto (ON) : Gouvernement de l'Ontario (Accédé 2019-09-07). https://www.ontario.ca/fr/lois/loi/90h07

5. ALPHA. Milestones and history: History of public health units in Ontario. Toronto (ON): Association of Local Public Health Agencies (Accédé 2019-09-07). https://www.alphaweb.org/ page/milestones

6. Ministère de la Santé et des Soins de longue durée. Normes de santé publique de l'Ontario : exigences relatives aux programmes, aux services et à la responsabilisation. Toronto (ON) : Ministère des soins de longue durée; 2018 (Accédé 2019-09-07). http://www.health.gov.on.ca/fr/pro/programs/ publichealth/oph_standards/docs/protocols_guidelines/ Ontario_Public_Health_Standards_2018_fr.pdf

7. Ministère de la Santé et des Soins de longue durée. Protocole concernant les plaintes relatives aux pratiques de prévention et de contrôle des infections, 2019. Toronto (ON) : Ministère des Soins de longue durée; 2019 (Accédé 2019-09-07). http://www.health.gov.on.ca/fr/pro/programs/ publichealth/oph_standards/docs/protocols_guidelines/ IPAC_Complaint_Protocol_2019_fr.pdf

8. Santé publique Ontario. Liste de vérification pour la prévention et le contrôle des infections $(\mathrm{PCl})$ dans le cadre de la pratique en cabinet et en clinique : Éléments essentiels. Toronto (ON): Santé publique Ontario; 2018 (Accédé 2019-09-07). https://www.publichealthontario.ca/-/ media/documents/checklist-clinical-office-core.pdf?la $=\mathrm{fr}$

9. Comité consultatif provincial des maladies infectieuses (CCPMI). Prévention et contrôle des infections pour la pratique en cabinet et en clinique. Toronto (ON) : Santé publique Ontario ; 2013 (Accédé 2019-09-07). https://www.publichealthontario.ca/-/media/documents/ bp-clinical-office-practice.pdf?la=fr

10. Provincial Infectious Diseases Advisory Committee. (2013). Pratiques exemplaires pour le nettoyage, la désinfection et la stérilisation du matériel médical dans tous les lieux de soins, 3e édition. Toronto (ON): Santé publique Ontario; 2013 (Accédé 2019-09-07). https://www.publichealthontario. $\mathrm{ca} /-/$ media/documents/bp-cleaning-disinfection-sterilizationhcs.pdf? $\mid \mathrm{a}=\mathrm{fr}$
11. Ministère de la Santé et des Soins de longue durée. Protocole concernant les déclarations relatives aux pratiques de prévention et de contrôle des infections, 2019. Toronto (ON) : Ministère de la Santé et des Soins de longue durée; 2019 (Accédé 2019-09-07). http://www. health.gov.on.ca/fr/ pro/programs/publichealth/oph_standards/docs/protocols_ guidelines/Infection_Prevention_and_Control_Disclosure_ Protocol_2019_fr.pdf

12. Statistics Canada. Health profile: health region - Ontario: public health units. Ottawa (ON) (Accessed 2019-0903). https://www12.statcan.gc.ca/health-sante/82-228/ search-recherche/lst/page.cfm?Lang=E\&GeoLevel=PR\&GEO $\mathrm{CODE}=35$

13. CDC. Healthcare-associated hepatitis $B$ and $C$ outbreaks $(>=2$ cases) reported to the Centers for Disease Control and Prevention (CDC) 2008-2017. Atlanta (GA): Centers for Disease Control and Prevention. https://www.cdc.gov/ hepatitis/outbreaks/pdfs/HealthcarelnvestigationTable.pdf

14. Centre d'expertise en retraitement des dispositifs médicaux. Démarche lors d'un bris de retraitement des dispositifs médicaux critiques et semi-critiques dans les établissements de santé. Quebec ( $\mathrm{QC})$ : L'Institut national de santé publique du Québec; 2016. https://www.inspq.qc.ca/sites/default/ files/publications/2196_bris_retraitement_dispositifs_ medicaux.pdf

15. Ministère de la santé et des services sociaux. Guide à I'intention des établissements: responsabilité et gestion d'un évènement indésirable lié au retraitement des dispositifs médicaux réutilisables. Quebec $(\mathrm{OC})$ : Gouvernement du Québec; 2013. http://publications.msss.gouv.qc.ca/msss/ fichiers/2013/13-209-02W.pdf

16. CDC. Steps for Evaluating an Infection Control Breach. Atlanta (GA): Centers for Disease Control and Prevention (Accédé 2019-09-07). https://www.colc.gov/hai/outbreaks/ steps_for_eval_ic_breach.html 